\title{
Igualdad de género, educación y trabajo: aproximación hacia la situación de Chile, Cuba y Finlandia
}

\section{Iskra Pavez Soto', Carmen Alfaro² y Laura Ochoa ${ }^{3}$ Universidad Bernardo O'Higgins}

\section{Artículo científico}

Material original autorizado para su primera publicación en el Journal de Ciencias Sociales, Revista Académica de la Facultad de Ciencias Sociales de la Universidad de Palermo.

\section{Recepción: 26-09-2018}

\section{Aceptación: 08-03-2019}

Resumen: El objetivo de este artículo es realizar una aproximación hacia la igualdad de género, la educación y el trabajo en tres países como Chile, Cuba y Finlandia. Se han seleccionado estos tres contextos porque tienen distintos modelos económicos y políticos. De este modo, se espera identificar su impacto en la igualdad de oportunidades para varones y mujeres. Para el desarrollo de este artículo se utiliza la metodología del análisis documental, a través de la yuxtaposición de determinados indicadores como son: Universalidad y gratuidad, formación del estudiantado, formación y calidad docentes, inversión en educación, desarrollo humano, participación de la familia en la educación, brecha de género, brecha salarial y participación laboral. Se concluye que el impacto ideológico que opera a través de un modelo económico específico puede afectar el trato igualitario y las oportunidades de modo concreto. Chile, por su parte, se destaca por los logros a nivel de acceso y cobertura educativa. Cuba, en tanto, presenta mejores indicadores de desempeño académico. Y Finlandia tiene altos indicadores de igualdad de género. Por todo lo anterior es que resulta de suma importancia considerar que algunas políticas se podrían replicar en nuestro país para de esta manera contribuir a la eliminación de las brechas de género.

Palabras claves: igualdad de género; educación; trabajo; modelo económico.

\footnotetext{
${ }^{1}$ Dra. en Sociología por la Universidad Autónoma de Barcelona, Experta en Políticas Sociales de Infancia por la Universidad Complutense de Madrid y Trabajadora Social por la UTEM. Actualmente es investigadora en el Centro de Investigación en Educación (CIE) de la Universidad Bernardo O'Higgins. Correo electrónico: iskra.pavez@ubo.cl

2 Estudiante de Doctorado en Educación en la Universidad Bernardo O'Higgins y Magíster en Ciencias Biológicas por la Universidad de Chile. Actualmente es académica de la Escuela de Tecnología Médica de la Universidad Bernardo O'Higgins,. Correo electrónico: carmenlalfaroc@gmail.com

${ }^{3}$ Estudiante de Doctorado en Educación en la Universidad Bernardo O'Higgins y Magister en Pedagogía Universitaria por la Universidad Mayor. Actualmente es Directora de la Escuela de Tecnología Médica de la Universidad Bernardo O’Higgins. Correo electrónico: laura.ochoa@ubo.cl
} 


\section{Equality of Gender, Education and Work: approximation to the situation in Chile, Cuba and Finland}

Abstract: The objective of this article is to make an approximation towards gender equality, education and work in three countries such as Chile, Cuba and Finland. These three contexts have been selected because they have different economic and political models, thus it is hoped to identify their impact on equal opportunities for men and women. For the development of this article, the methodology of documentary analysis is used, through the juxtaposition of certain indicators such as these: Universality and gratuity, student training, teacher training and quality, investment in education, human development, family participation in education, gender gap, wage gap and labor participation. It is concluded that the ideological impact that operates through an economic model can affect equal treatment and opportunities, specifically, Chile stands out for achievements in terms of access and educational coverage, Cuba has better academic performance indicators and Finland has high gender equality indicators. In view of the above, it is extremely important to consider that some policies could be replicated in our country in order to contribute to the elimination of gender gaps.

Key words: gender equality; education; work; economic model.

\section{Introducción}

El objetivo de este artículo es realizar una primera aproximación hacia la situación de igualdad de género, la educación y el trabajo en tres países que tienen distintos modelos económicos, como es el caso de Chile, Cuba y Finlandia, Cuba y Chile. En este sentido, nos proponemos analizar las brechas de género en educación y empleo pensando en las políticas públicas vigentes en cada territorio y cómo contribuyen a reducir las desigualdades. Considerando que los mandatos de género definen las funciones, los comportamientos, las actividades y los atributos que cada sociedad considera apropiados para varones y mujeres, esta socialización parte desde el hogar, pero en instituciones como las escuelas y los lugares de trabajo se siguen reproduciendo o, eventualmente, se pueden transformar.

Por ejemplo, es alarmante la desigualdad que aún persiste en Chile, basta con mirar brevemente algunos indicadores sobre brecha de género. Solo el $53 \%$ de las mujeres en comparación con el $85 \%$ de los varones en edad laboralmente activa (15 y 64 años) tiene un empleo; cifras diferentes a la media de países de la OCDE, donde el $62 \%$ de las mujeres y el $74 \%$ de los varones está insertos en el mercado laboral. Respecto a la brecha salarial, en Chile, las mujeres ganan un $23 \%$ menos que los varones en el trabajo remunerado, mientras que la media de los países de la OCDE es de un 12\% (OCDE, 2017). La diferencia de género en la participación de la fuerza laboral reduce los ingresos en un $20 \%$ per cápita. Esta proporción de pérdida de ingresos debida a la diferencia de género es la cuarta más alta en 
la OCDE y contribuye, en gran medida, a la desigualdad en general. Por indicadores como los recién comentados, Chile ocupa el puesto 63 de 145 países en el Índice Global de la Brecha de Género (Foro Económico Mundial, 2014). Si bien hoy en día las mujeres jóvenes chilenas estudian una mayor cantidad de años, en promedio, que los varones, no tienen las mismas oportunidades de conseguir un empleo o de recibir una remuneración equivalente a la de los varones que ocupan puestos similares; menos aún tienen posibilidades de optar a trabajos con cargos de liderazgo y jefaturas, tanto en organizaciones públicas como privadas.

El artículo se estructura de la siguiente manera: en la primera parte se describe el marco teórico referente al enfoque de género y su vinculación con educación y trabajo; luego, se presenta el contexto de los modelos económicos y políticos de los tres países estudiados. Posteriormente, presentamos los resultados y el debate, considerando una serie de indicadores. Finalmente, se entregan algunas conclusiones.

\section{Marco teórico: concepto de género}

Para Marcela Lagarde (1994, pp. 8-9), "el género es el conjunto de características sociales, culturales, políticas, psicológicas, jurídicas y económicas asignadas al sexo diferencialmente", un sistema de clasificación social de los seres humanos que tiene que ver con características corporales y pautas sociales de comportamiento. Dichas pautas son adquiridas y aprendidas a través de un complejo proceso de socialización que se vive a nivel individual y social. Por tanto, lo femenino y lo masculino son elementos que conforman un conjunto de oposiciones binarias y jerárquicas, que siguen la lógica de las clasificaciones vigentes en otros ámbitos de nuestra cultura, expresadas en conceptos como arriba-abajo, dentro-fuera, fuerte-débil, etc. (Lamas, 1999, p. 155, 164; 2007, p. 49). De acuerdo con la antropóloga Marta Lamas (1986), el rol de género, también denominado papel de género, se refiere al conjunto de normas y prescripciones dictadas tanto por la sociedad como por la cultura sobre el comportamiento femenino o masculino esperado (Lamas, 1986). Las sociedades realizan las designaciones de roles, funciones y prohibiciones a partir de las características biológicas, lo que depende del momento histórico en que se construyan dichas designaciones y, por tanto, el género está socioculturalmente situado (Abella et al., 2012).

Ciertamente, el concepto de género abre la puerta a una variedad de temas y cuestionamientos respecto a lo que significa ser niña o niño, mujer $u$ hombre en cada sociedad y sobre un determinado orden de género. Ahora bien, lo que nos interesa en este apartado es una definición de género aplicado al campo de la educación y el trabajo, que pasamos a ver a continuación. 


\subsection{Género y educación}

Al revisar la historia de las posibilidades de acceso a la educación por parte de las niñas y las mujeres se observa esta condición de menor privilegio y de subordinación a las labores domésticas (de atención, servicio y cuidado). Por ejemplo, desde el siglo XVI al XVII, Rousseau planteaba la inferioridad femenina. Posteriormente, en la Europa del siglo XVIII, se gestan las bases del sistema educativo moderno; allí surge la idea de que la educación no es necesaria para las niñas, ya que esto las sacaría de sus obligaciones domésticas. Luego, en el siglo XIX, las niñas y las mujeres se encuentran en un rol subordinado de procreación y cuidado de los hijos, idea promovida principalmente por la Iglesia Católica. No obstante, durante este siglo se fortalece la lucha feminista y se cuestiona la cultura patriarcal, las mujeres comienzan a acceder paulatinamente a espacios educativos. En el siglo XX se produjeron importantes cambios en cuanto a la situación de las niñas y las mujeres en la educación, su ingreso se consolida. Sin embargo, a inicios del siglo XXI aún persisten grandes brechas de género en cuanto al trato, los estereotipos y las trayectorias académicas (Reinoso \& Hernández, 2011).

La masiva incorporación de las niñas y las jóvenes a la educación formal durante las últimas décadas demuestra que es posible transformar y adecuar la institución escolar a las demandas sociales. No obstante, este acceso femenino no ha sido acompañado de un cambio profundo en las estructuras curriculares, en las metodologías, ni en la formación docente. La sola presencia de las niñas en las escuelas se ha interpretado como la igualdad de género deseable. Pero es necesario que valoremos críticamente la experiencia educativa que tienen las niñas y los niños en la escuela, que tomemos nota de las diferencias y las desigualdades, con el objetivo de mirar esa parte "invisible" que continúa subordinando un género a otro y, por lo tanto, impide la plena realización de las niñas y los niños, más allá de los estereotipos de género dominantes (Subirats, 2001, 2007; Subirats y Brullet, 2002; Tomé, 2002).

Algunas investigaciones sobre el sexismo en la escuela concluyen que la igualdad de acceso no implica la igualdad de trato, desde el punto de vista de la interacción en el aula, puesto que se han identificado claramente desigualdades de género: los niños tienen más posibilidades de recibir atención de parte de la figura docente, mientras que las niñas tienen menos probabilidades de ser escuchadas o tomadas en cuenta por parte de la figura docente (Subirats y Brullet, 1992, p. 11, 2002, p. 150). En otras palabras, las niñas tienen menos oportunidades de participar en el aula, requieren mayor aprobación adulta, piden menos la palabra, se adaptan a las normas porque son más dóciles (obedecen, son responsables), todo lo cual acaba por reproducir el protagonismo masculino en el ámbito público (los niños saltan, gritan, corren). 
Las expectativas que el profesorado tiene respecto a lo que se espera del rendimiento y de las prácticas de las niñas y los niños (cómo deben ser y qué deben hacer) es otro factor que incide en las dinámicas del aula respecto a las relaciones de género. Dado que las profesoras y los profesores representan figuras de autoridad y toma de decisiones en el proceso escolar, es indispensable considerarlos como un factor que ayuda a reproducir las jerarquías de género, o puede facilitar procesos de transformación y toma de conciencia (Subirats, 2007, pp. 139-140).

Además del género y la edad, la escolarización en que participan las niñas y los niños está determinada por las condiciones sociales y económicas donde se lleve a cabo el proceso educativo. Factores tales como la posición de clase social, el tipo de escuela (privada o pública) y los recursos de que dispone la familia influyen directamente en los resultados escolares y son claves para el proceso educativo infantil. Por otro lado, las jerarquías étniconacionales también juegan un papel importante respecto a las relaciones de género en la educación.

En el ámbito de la educación superior, la incorporación de las mujeres en las universidades y el incremento paulatino de su presencia a lo largo de los últimos siglos sobre todo en las últimas décadas-es, sin duda, un gran avance. Sin embargo, este proceso también está teñido por condiciones de desigualdad que dificultan su acceso, permanencia y movilidad (Buquet, 2016: 27). También en el campo de la investigación académica hay menor presencia de mujeres científicas que de varones (Beaudry y Larivière, 2016; Boyle et al, 2015). Por lo tanto, si la educación quiere brindar efectivamente igualdad de oportunidades, no solo debe garantizar el acceso y la cobertura, sino, también generar las mismas oportunidades de desarrollo a varones y mujeres, de manera que potencien sus habilidades y destrezas.

\subsection{Género y mercado laboral: la división sexual del trabajo}

Según Benería y Sen (1981), Benería (2005) y Molyneux (1994:147), la división sexual del trabajo se refiere a determinados roles, espacios y actividades asignadas y que se espera que realicen las mujeres y los varones diferenciadamente. De este modo, se construye una división jerárquica y binaria del trabajo, separando la producción de la reproducción. Si bien se basa en algunas diferencias biológicas entre los cuerpos de las mujeres y los varones, se trata principalmente de un ordenamiento que se fundamenta en las construcciones sociales, históricas y, sobre todo, económicas de cada sociedad. El reparto de labores productivas y reproductivas organiza los espacios donde se llevan a cabo dichas labores: un espacio público para la producción y uno privado para la reproducción. 
De acuerdo con Carrasquer et al (1998, p. 96), el trabajo reproductivo se caracteriza por:

no estar remunerado mediante un salario, ser un trabajo eminentemente femenino y permanecer invisible incluso a los ojos de las personas que lo llevan a cabo. El trabajo de la reproducción es la actividad a la que se dedican la gran mayoría de mujeres, a lo largo de su ciclo de vida, de manera total o parcialmente.

Pero el nivel económico de la familia es un factor que incide en la forma de distribución del trabajo reproductivo entre las niñas y mujeres del hogar; puesto que si existe el dinero suficiente se pueden contratar los servicios de otra mujer (joven, pobre o inmigrante) para que realice las labores de limpieza o cuidado.

La participación de las mujeres en la reproducción biológica de los seres humanos parece insoslayable, ya que es necesaria su presencia, al menos, en la gestación y el parto. Sin embargo, la reproducción social como tal, es decir, las tareas asociadas a la crianza y el cuidado, es una responsabilidad asignada socialmente a las mujeres mediante los procesos de socialización de género diferenciados. Por su parte, la reproducción de la fuerza de trabajo - llevada a cabo de manera gratuita y permanente- es un mecanismo que mantiene y, justamente, reproduce la mano de obra necesaria para que el sistema económico funcione. Las madres - $\mathrm{u}$ otras niñas y mujeres que cumplan ese rol- realizan un rol clave al brindar o no, apoyo afectivo y material durante la escolarización infantil y en la actividad laboral de los otros miembros del hogar (Benería, 1981, 2005; Saú, 1986, p. 39; Borderías y Carrasco, 1994).

Para Benería (1981, 2005, p. 85), las tareas asociadas a la reproducción se enseñan a las niñas como algo natural, propio de su género, pero ejercido con sutiles mecanismos de poder, control social y, en casos extremos, de manera obligatoria mediante el uso de la violencia. Como se piensa que son tareas naturales de las niñas y mujeres, se espera que las hagan por amor, de manera gratuita y sin esperar retribución económica alguna. Por las mismas razones tienen un escaso prestigio y valor social en nuestras sociedades (Borderías y Carrasco, 1994; Molyneux, 1994, p. 122; Hartman, 1994, p. 292). En el ámbito familiar se enseña y vigila que las niñas, jóvenes y mujeres aprendan y realicen este tipo de trabajos a través de los procesos de socialización de género. De esta manera, el hogar y el ámbito privado se convierten en el foco de trabajo para ellas, puesto que allí se desarrollan las actividades domésticas y de cuidado, asignadas socialmente, mientras se espera que niños y varones dominen las tareas asociadas al espacio público.

En síntesis, la división sexual del trabajo implica el mandato de una subordinación por parte de las niñas y las mujeres al espacio doméstico/privado, en las tareas reproductivas, como centro de su quehacer y realización personal, un espacio altamente invisibilizado. Por 
otro lado, este sistema dual prepara y exige a los niños y a los varones que se desarrollen en el ámbito público y en actividades productivas asociadas a su género, puesto que se les inculca que ellos son los responsables de mantener económicamente el hogar (Lamas, 1996, Benería, 2005; Saú, 2006).

\section{Contexto de los países estudiados}

En este apartado vamos a mostrar brevemente algunas características de los tres países estudiados, considerando sus modelos económicos y políticos.

\subsection{Chile}

El nombre oficial es República de Chile, se encuentra ubicado en el extremo sudoeste de América del Sur y tiene más de 17 millones de habitantes (INE, 2018). Posee un modelo de economía de libre mercado (neoliberal), donde el Estado tiene un rol subsidiario y regulador. Este modelo ha generado un importante crecimiento económico y ha permitido a la población un amplio acceso al consumo, situándolo entre los más altos de Latinoamérica; también se destaca que, después de la Dictadura Militar (1973-1989), ha logrado reducir la pobreza extrema manteniendo un escenario de estabilidad política. Sin embargo, tiene altos niveles de desigualdad en el acceso a educación, salud, vivienda y protección social. Por ejemplo, el coeficiente de Gini es de 0,51, considerado como uno de los más desiguales de América Latina y el Caribe, según reportes del Banco Mundial en 2015.

Respecto al modelo educativo, tras las reformas implementadas durante la Dictadura Militar se crearon tres tipos de establecimientos educativos: aquéllos que están en manos del Estado (municipalizados), mixtos (también llamados subvencionados) y privados. Los dos primeros tienen beneficios escolares, como alimentación escolar y subsidios estatales. Si bien el acceso y la cobertura escolar ha aumentado, la calidad de la educación pública sigue siendo baja si se compara con los grupos privilegiados (Saavedra, 2014).

En Chile, existe un $22 \%$ de jóvenes entre 15 y 29 años que no están ni educándose ni empleados o en formación (los llamados "NEET" o "NINI"), siendo que la media de los países OCDE bordea el 16\%. La mayoría de este grupo social pertenece a hogares pobres, son mujeres y tienen un bajo nivel de educación. En Chile, el 30\% de las mujeres entre 15 y 29 años son NEET, comparado con un 15\% de los varones de la misma edad. Empero, es preciso decir que este indicador mantiene un sesgo, debido a que el $30 \%$ de las mujeres consideradas como ni trabajando ni estudiando desarrollan labores de cuidado y servicio de otros (OCDE, 2015). Respecto al índice de brechas de género, Chile ocupa el lugar número 63 de 145 países, según el World Economic Forum (2015-2016). 


\subsection{Cuba}

Cuba es una isla ubicada en el Caribe y su población alcanza a los 11.475 .982 de habitantes. Sabido es que su modelo económico se basa en el ideal socialista, forjado durante la revolución de 1959 y su sistema político ha sido catalogado como una dictadura, por haber estado años bajo el mandato de la familia Castro. Recién este año se celebraron elecciones democráticas. Actualmente, Cuba tiene un coeficiente de Gini de 0,40, reportado para 2013 (Espina, 2014). El sistema educativo es público y está prohibida la existencia de la educación privada.

Tras la revolución, cambia la situación de las mujeres en la educación cubana, por ejemplo, se implementan medidas para erradicar el analfabetismo, que las afectaba de manera particular (Reinoso \& Hernández, 2011). El gobierno ha adherido a los instrumentos internacionales sobre la protección de los derechos de las mujeres y la disminución de las formas de discriminación por razones de género (Declaración de Beijing 1995, Cumbre del Milenio, 2000 y 2015 (Quaresma \& Ulloa, 2011). Cuba presenta una brecha de género del $74 \%$, ubicándose en el lugar número 27 del ranking internacional.

\subsection{Finlandia}

Finlandia es uno de los llamados países nórdicos, situado al noreste de Europa, integrante de la Unión Europea desde 1995 y con una población de 5.503.297 millones de habitantes.

En términos económicos, mantiene una estrategia de crecimiento a largo plazo de forma sostenida y se caracteriza por su desarrollo tecnológico. Tras la caída de la Unión Soviética, Finlandia sufrió una crisis económica, lo que la llevó a buscar alternativas para sortear esta situación, focalizando sus esfuerzos en lograr una robusta estructura educativa de manera de generar mano de obra cualificada. Este capital humano fortaleció las empresas logrando con ello un alto grado de competitividad; por ejemplo, ocupa el primer lugar en el índice de competitividad del World Economic Forum (Pampillón, 2008). Finlandia tiene un coeficiente de Gini de 0,26, considerado como bajo y presenta una brecha de género del $84.5 \%$, ubicándolo en el $3^{\circ}$ lugar a nivel mundial. Por lo anterior, es un país bastante igualitario, en general, y entre varones y mujeres, en particular.

Se caracteriza por contar con un Estado de bienestar fuerte, con una marcada orientación redistributiva y una importante intervención del sector público en materia económica y social. En el área de la educación el modelo económico Social Demócrata ha contribuido a generar un sistema comprehensivo y equitativo, orientado a garantizar 
oportunidades a los grupos más desfavorecidos y fundamentado en la participación directa del Estado en la provisión educativa (Verger et al, 2016).

El modelo educativo finés es reconocido internacionalmente, porque a menudo obtiene los primeros lugares en las pruebas estandarizadas de medición de la calidad. En este sistema la educación es obligatoria, gratuita y accesible para todos los y las niños/as, desde los 7 hasta los 16 años. Sus políticas han apuntado a desarrollar el concepto de equidad, que dice relación con la mantención de un sistema educativo que sea justo e inclusivo basado en la igualdad de oportunidades (Sahlberge, 2015). En Finlandia existe un alto compromiso con el aprendizaje de los estudiantes y no solo con los resultados que estos puedan obtener en una determinada prueba estandarizada.

\section{Metodología}

Este estudio se realizó desde la metodología de investigación de análisis documental a partir de ciertos casos seleccionados (Caballero et al, 2016, p. 40). Además, se pretende analizar las relaciones supuestas entre los fenómenos que pueden ser observados y que mantienen una relación entre sí, es decir, que comparten contextos; de este modo "explicarnos fenómenos y verificar si es posible generalizar a partir de esta explicación" (Senent, 2017, p. 3). Complementario con lo anterior, utilizamos el enfoque de resolución de problemas, a fin de relacionar o evidenciar diferencias o similitudes en los sistemas educativos y laborales de los tres países.

\section{Resultados}

A continuación, se presenta cada uno de los indicadores de análisis comparados de manera paralela en cada uno de los países estudiados (Chile, Cuba y Finlandia). Se realizó la yuxtaposición en forma textual de la información recopilada a partir de ciertos indicadores, tales como Universalidad y gratuidad; Formación del estudiantado; Formación y calidad docentes; Inversión en educación; Desarrollo humano; Participación de la familia en la educación; Brecha de género; Brecha salarial y Participación laboral.

\subsection{Universalidad y gratuidad}

Como primer punto partiremos diciendo que en Chile la educación se caracteriza por la segregación, es decir, los establecimientos privados son altamente elitistas, sus estudiantes provienen de los sectores con mayor holgura económica, contribuyen, tempranamente, a la desigualdad, debido a que las mejores condiciones financieras de estos 
establecimientos determinan mejores condiciones para el aprendizaje. En el caso de las entidades de educación superior, existen dos maneras de financiamiento público: aportes directos, según la oferta educativa, e indirectos, que subsidian a la demanda. Las universidades del Consejo de Rectores $(\mathrm{CRUCH})$ son las favorecidas con el primer sistema de financiamiento, de acuerdo con criterios establecidos. El aporte indirecto (AFI) corresponde a bonos por estudiantes sobresalientes en la Prueba de Selección Universitaria (PSU) otorgados a aquellas universidades con alta matrícula. Por otro lado, existen también los prestamos estudiantiles para pagos de colegiaturas (aranceles) provenientes del Fondo Solidario (estudiantes del $\mathrm{CRUCH}$ ) y del Crédito con Aval del Estado (CAE), dirigido a los alumnos de todas las Instituciones de Educación Superior, siempre que estén acreditadas (Hernández, 2017). En síntesis, en Chile no existe gratuidad universal, ya que esta condición está supeditada a la evaluación económica del estudiante, recayendo en la familia, el estudiante o su sostenedor un peso importante de los costos, lo que acaba generando situaciones de endeudamiento.

En Cuba la educación es de carácter universal y gratuita posicionándola como un eje del modelo de desarrollo cubano. Gran relevancia le ha dado el ministerio de educación al apoyo de los niños y las niñas en la primera infancia, esto se ha materializado con el Programa Educa a tu hijo (OEI, 2017).

En Finlandia la educación es gratuita, desde el preescolar hasta la universidad, y todas las instituciones educacionales pertenecen al Estado. Las únicas instituciones que pueden ser privadas corresponden a las Escuelas Vocacionales, es decir, aquellas que se dedican a la formación de un trabajo en particular y los Institutos Politécnicos. El modelo económico finlandés establece que el Estado no solo provea la educación, sino también la alimentación, los libros, el material educativo, servicios integrales de bienestar social y apoyo temprano a quienes lo necesiten, entre otras medidas. Por ley todo niño tiene derecho a este bienestar social y servicios de apoyo. Las políticas sociales de Finlandia han propiciado la igualdad de oportunidades, por lo que un $95 \%$ de los finlandeses y finlandesas se licencian de la educación secundaria para poder así optar a la educación superior (Sahlberg, 2015). Uno de los objetivos centrales de la política de educación finlandesa es darles a todos los ciudadanos y las ciudadanas las mismas oportunidades de acceso a la educación, sin discriminación de ningún tipo (Dirección Nacional Finlandesa de Educación, 2007).

\subsection{Formación del estudiantado}

En la prueba PISA, Chile ocupó el lugar 44 en la prueba de ciencias, el lugar número 42 en lectura y 48 en matemáticas (BBC, 2018), los datos de la prueba de PISA corresponden al año 2015. 
Cuba, por su parte, no participó en la última medición PISA, pero se puede destacar que en el Segundo Estudio Regional Comparativo y Explicativo (SERCE, 2004 al 2008) obtuvo los mejores resultados de América Latina y el Caribe, según indican portales adscritos al gobierno cubano, no precisando cifras (Torres, 2018).

Los estudiantes de Finlandia se encuentran dentro de los mejores resultados en la medición de los conocimientos que involucran las ciencias, matemáticas y de comprensión de lectura, en el último informe PISA entregado por la OCDE; ellos se encuentran en la quinta posición en la prueba de ciencias, en comprensión lectura se sitúan en la cuarta y en la doceava posición en matemáticas.

Un reciente informe de la UNESCO (2017) pone de relieve las desigualdades de género en la enseñanza de las ciencias, la tecnología, la ingeniería y las matemáticas (las llamadas "STEM"); aún persisten importantes desigualdades que dan cuenta de los obstáculos socioeconómicos y culturales que siguen mermando las posibilidades de las estudiantes (UNESCO, 2017). Según datos entregados en el último informe, en la enseñanza superior solo el $35 \%$ de los estudiantes matriculados en las carreras vinculadas con las STEM son mujeres. Actualmente, solo el $28 \%$ de los investigadores del mundo son mujeres. Según datos de la OCDE a pesar de que las diferencias por género en el caso de ciencias suelen ser pequeñas de media, en 33 países existe un mayor porcentaje de varones que de mujeres con un nivel excelente. Finlandia es el único país en el que las chicas tienen más probabilidades de alcanzar un nivel excelente que los chicos (PISA, 2016).

\subsection{Formación y calidad docente}

Si consideramos la formación del estudiantado, podemos observar que la realidad de Chile en este aspecto contrasta notoriamente con la realidad de los otros países. Hasta el año 1973, el país llevó a cabo una serie de medidas tendientes a fortalecer la educación desde la formación del profesorado. Durante el periodo de la Dictadura Militar, el país tuvo un considerable retroceso. Solo desde hace unas décadas se ha vuelto a poner en el tapete la calidad de la formación docente inicial y se han tomado medidas tendientes a mejorarla, sin obtener resultados sustanciales, que den cuenta del aprendizaje (Castro, 2015).

En Cuba existe un gran rigor en la formación y calidad del profesorado, toda vez que su objetivo central es la formación del profesor como mediador de la autonomía de sus estudiantes y la formación docente se comprende como una prioridad (Martín, 2015). La carrera dura cinco años y los docentes tienen una plaza de trabajo asegurada una vez que terminan sus estudios; la manera de designarles sus lugares de destino es de acuerdo con las necesidades de aquellas zonas deficitarias, en los cuales se destina a los mejores 
docentes, como una manera de elevar equitativamente la calidad de la educación a partir del desempeño profesional (Educar Chile, 2006).

El sistema educativo finlandés se cimienta sobre una sólida base en cuanto a la formación y calidad de sus docentes. Ellos tienen un nivel de formación sobresaliente, son seleccionados cuidadosamente y bajo estándares estrictos. Solo entre un $5 \%$ y $10 \%$ de los postulantes que desean entrar a estudiar pedagogía son aceptados. La selección de ellos incluye pruebas de lectura, sensibilidad artística, de dominios de instrumentos musicales y manejo comunicacional, entre otros. Además, para ejercer la profesión docente, los titulados deben contar con una maestría (Niemi, 2015). Los profesores finlandeses cuentan con un gran reconocimiento social dado su alto nivel profesional, esto no guarda relación con los sueldos, ya que este ítem equipara a los profesores de Europa en general, sino que más bien dice relación con el prestigio de su labor (Verger et al, 2016).

\subsection{Inversión en educación}

En cuanto a la inversión que proveen estos países, Chile y Cuba invierten respectivamente el $4,9 \%$ y el $12,8 \%$ de su Producto Interno Bruto (PIB), mientras que Finlandia no se encuentra dentro de los países que más invierte en educación, destina solo el 5,4\% (Banco Mundial, 2018). Comparativamente hablando, Finlandia es un país pequeño, con pocos habitantes, que ha sabido invertir de manera racional sin burocratizar sus procesos de manera de apuntar a los ejes centrales.

\subsection{Desarrollo humano}

El Índice de Desarrollo Humano (IDH) es un indicador que "mide el progreso conseguido por un país en tres dimensiones básicas del desarrollo humano: disfrutar de una vida larga y saludable, acceso a educación y nivel de vida digno" (PNUD, 2017). Según los resultados entregados por el Informe sobre Desarrollo Humano del Programa de Naciones Unidas para el Desarrollo (PNUD) en su versión 2015, las tres unidades comparativas se posicionan en los siguientes ubicaciones: Chile se posiciona en la ubicación 65. Junto a Argentina se consideran los únicos países latinoamericanos con un desarrollo muy alto y Cuba está en la ubicación 68; Finlandia se posiciona en el lugar número 11, siendo considerado como un país con muy alta calidad de vida (PNUD, 2015).

\subsection{Participación de la familia en la educación}

En cuanto a la participación de aquellos que conforman el contexto educativo familiar, en el caso de Chile no fue posible documentar una legislación específica al respecto, sobre 
las responsabilidades del Estado, el centro educativo y la familia, en otras palabras, no pudimos certificar el desarrollado e institucionalización de una instancia de participación activa de padres en el interior del aula.

En el caso de Cuba, existe un compromiso que es señalado por la Constitución de la República en el denominado Código de la Familia, allí se establecen responsabilidades entre la familia y el Estado que permiten la coordinación de las influencias educativas en una sola dirección. En Finlandia los padres se transforman en participantes activos del proceso de enseñanza-aprendizaje, colaborando en el interior de las escuelas como asistentes que apoyan la labor docente, cuyos cursos están formados por grupos multigrados (Bolaños, 2012).

\subsection{Brecha de género}

Si nos desplazamos al ámbito de la equidad, al comparar los resultados obtenidos en el último informe de brecha de género desarrollado por el Foro Económico Mundial, Chile se posiciona en el puesto 63, Cuba en la posición vigesimoquinta y Finlandia en el tercer lugar.

\subsection{Brecha salarial}

Si se considera la brecha a nivel salarial, en Chile el reflejo de esta paupérima posición se evidencia en la gran disparidad en relación con las remuneraciones y la baja presencia de mujeres en altos cargos en comparación con los varones. Tanto Cuba como Finlandia cierran su brecha en relación con el logro educativo (Informe GET, 2016).

\subsection{Participación laboral}

Con relación a la participación laboral, tanto Chile como Cuba se encuentran por debajo de los países desarrollados de la OCDE (61\%), mientras que en el país nórdico alcanza casi a la paridad. Es importante mencionar que pensar en la igualdad es considerar a las mujeres como parte de la economía inteligente, ya que esta puede aumentar la eficacia económica y contribuir a las mejoras en materia de desarrollo.

En la Tabla № 1 se pueden apreciar, a modo de síntesis, cómo se relacionan los indicadores de cada uno de los países estudiados:

Tabla 1. Síntesis de la igualdad de género, educación y trabajo en Chile, Cuba y Finlandia 


\begin{tabular}{|c|c|c|c|}
\hline País & Chile & Cuba & Finlandia \\
\hline $\begin{array}{l}\text { Modelo } \\
\text { económico y } \\
\text { político }\end{array}$ & $\begin{array}{l}\text {. Neoliberal } \\
\text { Estado subsidiario } \\
\text { y regulador. }\end{array}$ & $\begin{array}{l}\text { Socialista } \\
\text { Solidaridad }\end{array}$ & $\begin{array}{l}\text { Social } \\
\text { Demócrata. } \\
\text { Estado de } \\
\text { Bienestar }\end{array}$ \\
\hline $\begin{array}{l}\text { Financiamiento } \\
\text { de la educación }\end{array}$ & $\begin{array}{l}\text { Público } \\
\text { Privado } \\
\text { - Mixto } \\
\text { (subvencionado) }\end{array}$ & $\begin{array}{l}\text { Universal } \\
\text { Gratuita } \\
\text { Pública. }\end{array}$ & $\begin{array}{l}\text { - Gratuita } \\
\text { Pública. } \\
\text { - Excepción de } \\
\text { Escuelas } \\
\text { Vocacionales e } \\
\text { Institutos } \\
\text { Politécnicos. }\end{array}$ \\
\hline $\begin{array}{l}\text { Singularidad del } \\
\text { sistema } \\
\text { educativo }\end{array}$ & $\begin{array}{l}\text { Amplio acceso y } \\
\text { cobertura } \\
\text { Segregación entre } \\
\text { establecimientos } \\
\text { públicos, mixtos y } \\
\text { privados (élites). }\end{array}$ & $\begin{array}{l}\text { Amplio acceso } \\
\text { No existe educación } \\
\text { privada } \\
\text { Énfasis en la } \\
\text { primera infancia. }\end{array}$ & $\begin{array}{l}\text { Equidad } \\
\text { - Justicia } \\
\text { - Inclusión } \\
\text { - Igualdad de } \\
\text { oportunidades }\end{array}$ \\
\hline $\begin{array}{l}\text { Formación y } \\
\text { calidad docente }\end{array}$ & $\begin{array}{l}\text { Deficitaria } \\
\text { Medidas } \\
\text { insuficientes } \\
\text { Reformas } \\
\text { incipientes. }\end{array}$ & $\begin{array}{l}\text { Prioritaria } \\
\text { Rigurosa } \\
\text { Rol mediador de } \\
\text { autonomía } \\
\text { estudiantil. }\end{array}$ & $\begin{array}{l}\text { - Nivel destacado } \\
\text { - Selección estricta } \\
\text { - Alta formación. }\end{array}$ \\
\hline Obligatoriedad & $\begin{array}{l}\text { De } 4 \text { a } 18 \text { años } \\
\text { De prekinder a } 4^{\circ} \\
\text { año de Enseñanza } \\
\text { Media. }\end{array}$ & $\begin{array}{l}\text { De } 6 \text { a } 14 \text { años } \\
\text { De educación } \\
\text { primaria a } \\
\text { secundaria básica. }\end{array}$ & $\begin{array}{l}\text { De } 7 \text { a } 16 \text { años } \\
\text { De educación } \\
\text { básica a } \\
\text { educación } \\
\text { secundaria. }\end{array}$ \\
\hline $\begin{array}{l}\text { Inversión en } \\
\text { Educación }\end{array}$ & $\begin{array}{l}\text { 4,9\% de su } \\
\text { Producto Interno } \\
\text { Bruto (PIB) }\end{array}$ & $\begin{array}{l}12,8 \% \text { de su } \\
\text { Producto Interno } \\
\text { Bruto (PIB) }\end{array}$ & $\begin{array}{l}5,4 \% \text { de su } \\
\text { Producto Interno } \\
\text { Bruto (PIB) }\end{array}$ \\
\hline
\end{tabular}




\begin{tabular}{|c|c|c|c|}
\hline $\begin{array}{l}\text { Participación } \\
\text { de la familia en } \\
\text { la educación }\end{array}$ & $\begin{array}{l}\text { Sin legislación } \\
\text { · Libertad en cada } \\
\text { centro educativo }\end{array}$ & $\begin{array}{l}\text { Legislación señala } \\
\text { responsabilidades } \\
\text { entre la familia y el } \\
\text { Estado } \\
\text { Coordinación de las } \\
\text { influencias } \\
\text { educativas }\end{array}$ & $\begin{array}{l}\text { · Legislación } \\
\text { señala participan } \\
\text { activa } \\
\text { · Colaboración con } \\
\text { centros } \\
\text { educativos }\end{array}$ \\
\hline $\begin{array}{l}\text { Posición en la } \\
\text { brecha de } \\
\text { género }\end{array}$ & . $63^{\circ}$ & $27^{\circ}$ & $\cdot 3^{0}$ \\
\hline $\begin{array}{l}\text { Participación } \\
\text { laboral femenina }\end{array}$ & . 57,9\% & $51,1 \%$ & $74,3 \%$ \\
\hline $\begin{array}{l}\text { Participación } \\
\text { laboral } \\
\text { masculina }\end{array}$ & $\cdot 80,1 \%$ & 79,1 & . $77,0 \%$ \\
\hline
\end{tabular}

Fuente: Elaboración propia.

\section{Discusión}

Con todo, podemos verificar que Chile aún dista mucho del desarrollo que ha logrado Finlandia e incluso Cuba, considerando las vicisitudes económicas que ha sorteado ese país. El caso de Finlandia y Cuba resulta ser más bien emblemático, ya que podemos identificar variados elementos que configuran el desarrollo de iniciativas que han permitido trabajar en post de la equidad de género y de acciones que no impongan limitaciones externas o que impacten desigualmente a varones y mujeres. Lo primero y fundamental en estos dos países es la convicción de que la educación es un derecho, que resguarda el Estado, luego de ello, su actuar en coherencia con las acciones que llevan a cabo para establecerla como tal: la gratuidad y la universalidad. Esta sola medida previene la segregación social y la pérdida de autonomía de las personas, dado que no habrá impedimentos económicos para la decisión de estudiar y la elección acerca de qué estudiar al término de la enseñanza secundaria. Con ello, el Estado resguarda la permanencia de los y las jóvenes en el sistema educativo y su progreso, a partir de una formación integral y de calidad. En torno a esta idea, como eje 
central, ellos atribuyen a la formación docente y a la participación de la familia un rol preponderante en la educación de niños y niñas.

Chile, por su parte, aún presenta importantes desafíos al respecto. Sin embargo, incluso cuando, tanto Cuba como Finlandia destacan en las evaluaciones internacionales respecto a estas áreas, no han logrado sortear la brecha de género en este ámbito. Cuba y Finlandia han promovido el aprendizaje significativo de sus estudiantes con excelentes logros, han dado importancia a la alfabetización científica y tecnológica cimentada en las ciencias y las matemáticas, considerando que estas disciplinas aportan conocimiento científico para apoyar el avance en ciencias, tecnología y salud del ser humano. Además, se proyectan como las disciplinas en las que se fundamentan las carreras del futuro.

Es importante detenernos en este punto, debido a que las brechas en educación vinculada con las llamadas "STEM" podrían dar cuenta de procesos de socialización y aprendizaje inicial que promueve en los niños y las niñas la adopción de constructos socioculturales que favorecen los estereotipos de género y la asignación de roles asociados. Esto influye sobre su identidad, el comportamiento, sus convicciones y sus decisiones. Es decir, se sigue reproduciendo la división sexual del trabajo. A las niñas se les educa atribuyéndoles importancia a un "rol de servicio y cuidado", desvalorizando sus capacidades para lo relativo a las STEM, impregnándolas de la idea que son temas "masculinos" y de que la capacidad femenina en este ámbito es inferior a la de los varones. Aun cuando no existen evidencias científicas que sustenten esta creencia, la transferencia histórica de estereotipos de género en la educación formal ha impactado negativamente en las oportunidades de las niñas y las mujeres para acceder a mejores opciones salariales, de empleos y puestos de poder. Es un hecho de la realidad la feminización de ciertas carreras que se asocian a los roles históricamente asignados a las mujeres (carreras del área de la salud, educación, social, etc.), en desmedro de su incorporación a carreras que se vinculan con las STEM y que ofrecen mejores condiciones salariales en el espacio laboral y otorgan un mayor estatus simbólico (Comunidad Mujer, 2017).

Es así como se puede mencionar que: "datos del último informe PISA revelan que, a los 15 años, chicos y chicas ya tienen visiones distintas sobre sus capacidades y ellas están menos satisfechas con la vida que ellos. Los padres y madres también tienen diferentes expectativas para sus hijos e hijas dependiendo de si son varones o mujeres. Por ejemplo, en Chile, el 50\% de los padres de chicos de 15 años esperan que trabajen en áreas STEM ciencia, tecnología, ingeniería y matemáticas- mientras que para las hijas solo lo desean el $18 \%$ de los progenitores".

Una iniciativa desarrollada en Finlandia, respecto de la educación sin estereotipos, es el proyecto TASUKO, que tiene como foco la igualdad y conciencia de género en la formación 
docente. Eliana Lahelma (2011), responsable a cargo este proyecto, señala que "no obstante los avances que Finlandia ha dado en relación con el tema de igualdad de género, aún se requiere desarrollar un trabajo que permita la conciencia de género de manera específica a nivel de la formación del profesorado, ya que ellos son actores de suma relevancia en la perpetuación o modificación de los estereotipos de género".

\section{Conclusiones}

A diferencia del sistema educacional chileno, el sistema finlandés y el cubano no han experimentado reformas educacionales mercantilistas que promueven la competencia, la privatización de las escuelas públicas y el énfasis en las pruebas estandarizadas de alto impacto para los estudiantes. En Chile, el modelo económico neoliberal favorece el individualismo, alimentando la desigualdad social al no concebir la educación, el trabajo y la familia como un sistema integrado y a las mujeres y los varones como seres integrales que desarrollan sus vidas en todos esos ámbitos. Tanto en Cuba como en Finlandia las políticas educativas están concebidas de manera de generar igualdad de oportunidades.

Hoy en día se reconoce que el modelo de expansión de la cobertura educativa, vigente desde las últimas cuatro décadas, ha entrado en crisis, debido al deterioro de la calidad de la oferta educativa. Este deterioro en la calidad de la educación juega un papel crucial, configurándose como un obstáculo en asegurar un desarrollo productivo con equidad $y$, en consecuencia, brindar a todos los sectores sociales y, por tanto, a las mujeres y los varones, el acceso a un estándar mínimo de calidad durante su formación.

No obstante lo anterior, debemos convenir en que es un hecho ampliamente reconocido que la expansión del sistema de educación formal benefició de manera particular a las mujeres y, complementariamente, que el cambio más evidente en su condición social se asocia en su acceso masivo a la educación, aunque, en el caso particular de Chile, específicamente en la educación superior, los varones acceden en mayor proporción a los planteles estatales o tradicionales antes que a los privados. No sucede lo mismo con las mujeres, quienes vieron favorecido su acceso a expensas de las instituciones privadas.

Es importante preguntarse qué aprenden ellas sobre sí mismas y su futuro papel en la sociedad, por qué continúan orientándose hacia campos profesionales "tradicionalmente femeninos", qué efectos tiene el contexto educativo, en el desarrollo de su identidad, autoestima y proyecto de vida, cuáles son los mensajes que se transmiten a través del currículum formal y oculto. En definitiva, profundizar en los procesos y mecanismos manifiestos y sutiles a través de los cuales la educación transmite un conjunto de valores y 
expectativas diferenciales según género, que posteriormente se traducirán en menores oportunidades para las mujeres.

Finalmente, podemos concluir que los antecedentes recopilados nos entregan evidencias que pueden ser replicadas para que Chile continúe en la senda que se requiere para disminuir y, en un futuro no muy lejano, eliminar las brechas de género. El impacto ideológico que opera a través de un modelo económico puede afectar el trato igualitario y las oportunidades que derivan de ello. Sin embargo, el respeto a los derechos humanos, a los derechos de los niños y las niñas debiera estar por sobre esto.

\section{Referencias bibliográficas}

Abella, P., Páez, J. \& Seidel, B. (2012). La construcción de la identidad de género en la primera infancia: tensiones entre la resistencia y el cambio. (Tesis de Doctorado en Educación.). Pontificia Universidad Javeriana, Bogotá, Colombia.

Benavente, M. y Valdés, A. (2014). Políticas públicas para la igualdad de género: un aporte a la autonomía de las mujeres. Libros de la CEPAL, № 130 (LC/G.2620-P), Santiago: Comisión Económica para América Latina y el Caribe.

Beaudry, C. y Lariviere, V. (2016). Which gender gap? Factors affecting researchers' scientific impact in science and medicine. Research Policy, 45(9), 1790-1817, DOI: http://dx.doi.org/10.1016/j.respol.2016.05.009.

Benería, L. (2005). Género, desarrollo y globalización. Por una ciencia económica para todas las personas. Barcelona: Editorial Hacer.

Benería, L. y Sen, G. (1981) Accumulation, reproduction and women's role in economic development, Boserup Revisited, Signs, 7(2), 279-298.

Bolaños, D. (2012). Educación rural en Finlandia: experiencias para México. CPU-e, Revista de Investigación Educativa, 15,140-154.

Borderías, C. y Carrasco, C. (1994). Introducción. Las mujeres y el trabajo: aproximaciones históricas, sociológicas y económicas. En C. Borderías, C. Carrasco. \& C. Alemany (Comps.), Las mujeres y el trabajo. Rupturas conceptuales (pp. 18-92). Barcelona: Icaria-Fuhem.

Boyle, P., Smith, L., Cooper, N., Williams, K. \& O'Connor, H. (2015). Gender balance: Women are funded more fairly in social science. Nature, 525, 181-183, DOI: doi:10.1038/525181 a

Buquet, A. (2016). El orden de género en la educación superior: una aproximación interdisciplinaria. Revista Nómadas, 44, 27-43. 
Caballero, A., Manso, J., Matarranz, M., \& Valle, J. (2016). Investigación en Educación Comparada: Pistas para investigadores noveles. Revista Latinoamericana de Educación, 9, 39-56.

Carrasquer, P., Torns, T., Tejero, E. y Romero, A. (1998). El trabajo reproductivo. Papers, 55, 95-114.

Castañeda, I., Astraín, M., Sarduy, C. \& Rodríguez, A. (1999). Algunas reflexiones sobre el género. Revista Cubana Salud Pública, 25(2),129-142.

Castro, F. (2015). Formación de Profesores en Chile: Una mirada crítica ante la encrucijada histórica de los cambios educacionales. Revista Praxis Educativa, 19 (1),12-19.

Espina, M. (2014). Desigualdad social y retos para una nueva institucionalidad democrática en la Cuba actual. Espacio Laical, 2,101-104.

Hartmann, H. (1994). Capitalismo, patriarcado y segregación de los empleos por sexos. En C. Borderías, C. Carrasco \& C. Alemany (Comps.).,Las mujeres y el trabajo. Rupturas conceptuales (pp. 253-294). Barcelona: Icaria-Fuhem.

Lagarde, M. (1994). Género e Identidades: metodología de trabajo con mujeres. Quito: FUNDETEC-UNICEF.

Lahelma, E. (2011). Gender Awareness in Finish Teacher Education: An Impossible Mission? Education Inquiry, 2(2), 263-276.

Lamas, M. (1996). La antropología feminista y la categoría de "género". Nueva Antropología, 8 (30), 173- 198.

Lamas, M. (1999). Usos, dificultades y posibilidades de la categoría género. Papeles de Población, 21, 147-178

Lamas, M. (2007). Algunas reflexiones relativas al derecho a decidir sobre el propio cuerpo En J. Astelarra (Coord.), Género y cohesión social (pp. 43-51). Madrid: Fundación Carolina.

Martín, D. (2015). La formación docente universitaria en Cuba: Sus fundamentos desde una perspectiva desarrolladora del aprendizaje y la enseñanza. Estudios pedagógicos, XLI (1), 337-349.

Molyneux, M. (1994). Más allá del debate sobre el trabajo doméstico. En C. Borderías, C. Carrasco \& C. Alemany (Comps.), Las mujeres y el trabajo. Rupturas conceptuales (pp. 111-150). Barcelona: Icaria-Fuhem.

Niemi, H. (2015). Desarrollo profesional docente en Finlandia: Hacia un enfoque más holístico. Psychology, Society and Education, 7(3), 387-404. 
Ocegueda, J., Miramontes, M., Moctezuma, P.\& Mungaray, Alejandro. (2017). Análisis comparado de la cobertura de la educación superior en Corea del Sur y Chile: una reflexión para México. Perfiles educativos, 39(155), 141-159.

Pampillón, R. (2008). El modelo Nórdico. Revista de Economía Mundial, 18,155-165.

Quaresma, D. \& Ulloa, O. (2011). Masculinidades en Cuba: legitimación de una dimensión de los estudios de género. Revista de Estudios Sociales, 42,93-103.

Reinoso, I. \& Hernández, J. (2011). Las perspectivas de género en la educación. Cuadernos de Educación y Desarrollo, 3 (28), 34-56.

Saavedra, E. (2014). El Modelo Económico-Político de Chile: Desarrollo Institucional en la Encrucijada. Revista Economía y política, 1(1), 117-148.

Senent, J. M. (2017). Educación Comparada: Concepto, Evolución, Metodología y Fuentes. Dossier docente. Doctorado en Educación. Universidad de Valencia.

Rubilar, F. (2015). Formación de Profesores en Chile: Una mirada crítica ante la encrucijada histórica de los cambios educacionales. Praxis educativa, 19(1), 12-19.

Saú, V. (1993[1986]). Ser mujer: el fin de una imagen tradicional. Barcelona: Icaria.

Saú, V. (2006). Reproducción social de las identidades de género: de niñas a mujeres. En A. Rincón (Coord.), Congreso Internacional SARE 2005: "Niñas son, mujeres serán" (pp. 16-20). Bilbao: Emakunde/Instituto Vasco de la Mujer.

Shalberge, P. (2015). Un sistema escolar modelo. Finlandia demuestra que la equidad y la excelencia pueden coexistir en la educación. Pensamiento Educativo. Revista de Investigación Educacional Latinoamericana, 52 (1),136-145.

Subirats, M. (2001). ¿Qué es educar? De la necesidad de reproducción a la necesidad de cambio. En X, Rambla y A., Tomé (Eds), Contra el sexismo: coeducación y democracia en la escuela (pp. 156-180). Madrid: Editorial síntesis.

Subirats, M. (2007). De la escuela mixta a la coeducación: la educación de las niñas, el aprendizaje de la subordinación. En A. Vega Navarro (Coord.), Mujer y educación: una perspectiva de género (pp. 137-148). Málaga: Ediciones Aljibe.

Subirats, M. y Brullet, C. (1992). Rosa y azul. La transmisión de los géneros en la escuela mixta. Serie Estudios № 19. Madrid: Ministerio de Asuntos Sociales-Instituto de la Mujer.

Subirats, M. y Brullet, C. (2002). Rosa y azul. La transmisión de los géneros en la escuela mixta. En A., Gonzalez y C. Lomas (Coord.), Mujer y educación. Educar para la igualdad, educar desde la diferencia (pp. 133-168). Barcelona: Editorial Graó del Rif. 
Tomé, A. (2002). Luces y sombras en el camino hacia una escuela coeducativa. En A. González y C. Lomas (Coord.), Mujer y educación. Educar para la igualdad, educar desde la diferencia (pp. 169-182). Barcelona: Editorial Graó del Rif.

Tomé, A. (2007). Las relaciones de género en la adolescencia. En A. Vega Navarro (Coord.), Mujer y educación. Una perspectiva de género (pp. 117-136). Málaga: Ediciones Aljibe.

Tonon, G. (2011). La utilización del método comparativo en estudios cualitativos en ciencia política y ciencias sociales: Diseño y desarrollo de una tesis doctoral. Revista de Temas Sociales, 15(27), 1-12.

Verger, A., Zancajo, A., \& Fontdevilla, C. (2016). La economía política de la privatización educativa: políticas, tendencias y trayectorias desde una perspectiva comparada. Revista Colombiana de Educación, 70, 47-78.

\section{Documentos de prensa publicados electrónicamente}

BBC Mundo. (2018). Pruebas PISA: ¿cuáles son los países que tienen la mejor educación del mundo? ¿Y cómo se ubica América Latina? [en línea]. Disponible en http://www.bbc.com/mundo/noticias-38211248 [consulta: 15 de enero 2018].

\section{Documentos de organismos públicos de ámbito nacional}

Dirección Nacional Finlandesa de Educación (2007). Sistema escolar. Helsinki: Dirección nacional finlandesa de educación.

Educar Chile. (2006). Educación y evaluación docente en Cuba. [en línea] Disponible en http://www.educarchile.cl/ech/pro/app/detalle?ID=106595 [consulta: 14 de enero 2018].

Instituto Nacional de Estadísticas. (2018). Estadísticas Demográficas y Vitales. Santiago: INE.

\section{Documentos de organismos no gubernamentales de ámbito nacional}

Comunidad Mujer. (2016). Resumen Ejecutivo Informe GET, Género, Educación y Trabajo: la brecha persistente. Primer estudio sobre la desigualdad de género en el ciclo de vida. Una revisión de los últimos 25 años. Santiago: Comunidad mujer.

Comunidad Mujer (2017). Estadísticas de género. [en línea] Disponible en www.comunidadmujer.cl/biblioteca.../wp.../12/BOLETIN-42-DIC-2017-url_vf.pdf [consultado: 20 de enero 2018]. 


\section{Documentos de organismos internacionales}

Foro Económico Mundial. (2017). The Global Gender Gap Report. Ginebra: Foro Económico Mundial.

ONU Mujeres. (2015). Declaración y Plataforma de Acción de Beijing. Nueva York: ONU Mujeres.

UNESCO. (2015). América Latina y el Caribe: Revisión Regional de la Educación 2015 para todos. Santiago: UNESCO.

UNESCO. (2017a). Cracking the code: Girls' and women's education in science, technology, engineering and mathematics. Paris: UNESCO.

UNESCO. (2017b). La educación transforma vidas. Paris: UNESCO.

\section{Documentos de organismos internacionales publicados electrónicamente}

Banco Mundial. (2018). Gasto público en educación. [en línea]. Disponible en https://datos.bancomundial.org/indicador/SE.XPD.TOTL.GD.ZS?locations=CLCU-FI [consulta: 28 de mayo 2018].

ECOSOC. (2012). Informe del Consejo Económico y social para 2012. [en línea] Disponible en http://www.un.org/es/comun/docs/?symbol=A/67/3 [consulta: 18 de enero 2018].

PNUD. (2015). Informe sobre Desarrollo Humano 2015. Trabajo al servicio del desarrollo humano. [en línea] Disponible en http://www.undp.org/content/undp/es/home/librarypage/hdr/2015-humandevelopment-report.html [consultado: 18 de enero 2018].

Programa de las Naciones Unidas para el Desarrollo. (2018). Informe Nacional de Desarrollo Humano [en línea]. Disponible en http://desarrollohumano.org.gt/ [consulta: 28 de mayo de 2018].

Organización de Estados Iberoamericanos. (2018). Educación Inicial. Experiencias no Escolarizadas. Programa Educa a tu hijo. [en línea]. Disponible en http://www.oei.es/historico/inicial/cubane.htm [consultado: 15 de enero 2018].

OCDE (2017). Evaluaciones de Políticas Nacionales de Educación. Educación en Chile. [en línea]. Disponible en: https://www.oecd-ilibrary.org/education/revision-de-politicasnacionales-de-educacion_1990021x [consulta: 28 de mayo de 2018]. 
OCDE. (2016). PISA 2015 Resultados Clave. [en línea]. Disponible en https://www.oecd.org/pisa/pisa-2015-results-in-focus-ESP.pdf [consultado 28 de mayo 2018].

OECD. (2015). Todos Juntos: ¿Por qué reducir la desigualdad nos beneficia? [en línea]. Disponible en https://www.oecd.org/chile/OECD2015-In-It-Together-HighlightsChile.pdf [consultado: 28 de mayo 2018].

OMS. (2018). El género. [en línea]. Disponible en http://www.who.int/topics/gender/es/ [consulta: 18 de enero de 2018].

ONU Mujeres. (2015). La Plataforma de Acción de Beijing cumple 20 años. [en línea]. Disponible en http://beijing20.unwomen.org/es/in-focus/education-and-training [consultado 15 de enero 2018]. 